\title{
A Probabilistic Model for Object based Foreground Segmentation from Multimedia
}

\author{
Mr. P. Thangaraju, M.Sc., MCA, M. Phil., PGDCA ${ }^{1}$, Mrs. R. Nanthini., M.Sc., B.Ed., ${ }^{2}$ \\ Computer Science, Bishop Heber College, Bharathidasan University ${ }^{1,2}$
}

\begin{abstract}
The image processing is used for video segmentation, the inherent unreliability of frequently results in corrupted video packets and jitter during video playback. Such a deviation is especially pronounced in processing of videos due to the fading and multipath interference impediments in image processing. Algorithm based video transmission provides reliability at the cost of severely increased delay arising from frequent retransmissions in case of packet error over constraints from session and application layers for in-sequence delivery. These delays are substantially worsened in low SNR (signal-to-noise power ratio) environments. Multiple connections, which efficiently utilize the bandwidth of the channel, have been demonstrated to yield significantly improved quality of video segmentation over image processing while enhancing the reliability of video delivery compared to methods. It proposes new scalable classified adaptive bit rate video coding over heterogeneity atmosphere for reliable video segmentation while reducing the delay and jitter of video foreground subtraction.
\end{abstract}

Keywords: Bandwidth, Error Control, Overhead, SNR, Prioritization, Video Segmentation, Foreground Subtraction.

\section{INTRODUCTION}

Video co-segmentation is to detect the main object in videos and to delineate it from the contextual in all surrounds. Video object segmentation is a well-researched problem in the computer vision public and is a prerequisite for a variety of high-level vision applications, including content based video retrieval, video summarization, and activity sympathetic and targeted content replacement. Both fully automatic methods and methods requiring manual initialization have been proposed for cinematic object subdivision. Despite many attempts [3]-[9], the problem remains problematic or even ill-defined. In those methods based on a single image, conventional mid-level visual cues to foreground circumstantial assignment such as convexity and parallelism are used [3]-[6]. However, most proposed demonstrations are still too local and bottom-up to handle the complex contradiction in natural images. They were usually established solely on line images, with a few exclusions [4], [5].However, the communication systems deployed for the transport of the video data of these outdoor systems are expensive [2]. Broadband allow real-time segmentation of video footage from inside transit vehicles, providing first responders with the actionable intelligence they need to respond effectively to any emergency situation [1].

In this work, exploit the possibility of path diversity to use of streams lost packets and use forwarding of multiple packets from different streams. This approach avoids streams contending in the most congested region and achieves packets delivery before playing deadline. This performance metrics of interest is the average video streams delay, the video streams throughput, the video streams loss and the packet delivery ratio under proposed algorithm.

\section{RELATED WORK}

Many approaches to using Forward Error Control (FEC) algorithms have been extensively analyzed in literature. In [4] a method of using FEC codes called Linear Increase Multiplicative Decrease with History (LIMD/H) to stream Internet video is discussed. The method uses FEC codes to achieve robust and efficient Internet video segmentation and multicast consistent with the existing Internet architecture. This approach used history of packet losses to measure congestion levels in the network and incorporated FEC to formulate an algorithm that converts the prioritized stream of bits into an un-prioritized stream of bits [4].

From the simulation results presented in [4], it was observed that LIMD/H offers an approach that better tolerates variations of packet loss in multimedia transmissions over the Internet. However, because LIMD/H looks up the history of packet loss before adjusting the transmission rate in the network, this causes slowness in responding to changing network conditions. The approach described in this paper proposes a method that uses queue length at the Access Point as a measure for congestion thereby ensuring a faster response time while dynamically responding to changing network dynamics. Another approach that combines FEC with prioritization is that described in [5]. The authors investigated the effects of adapting interleaved packet-level FEC according to packet transmission properties to address issues of delay introduced during correction of errors in video streams at the wireless channel [5]. 
The original packets where further prioritized according to a packets assumed importance [5] and given additional FEC protection. The interleaving method introduces delays in the network because packets often have to be reordered. The proposed algorithm corrected packet errors due to wireless channel error using a Static FEC mechanism. This means there is a constant high FEC overhead in the network regardless of wireless channel conditions. Such a setup will perform poorly when the wireless network deteriorates.

The authors efficiently compute the number of FEC packets so as to not hamper TCP performance in the network [6]. Although the authors in [6] focus mainly on the bandwidth trade-off between bandwidth consumed by FEC packets and that used by TCP, the adaptive FEC mechanism used has similarities to the proposed mechanism in this paper in ensuring fairness of network resource usage. However, the approach described in this paper not only makes the addition of FEC redundancies in such a way that the loss probability of packets in the network is reduced but also improves delivered video quality by adapting the level of FEC in the network based on current network channel conditions.

\section{VIDEO SEGMENTATION DESIGN SCHEDULING}

In this work the video streams from $\mathrm{n}$ VSs to schedule with the combination of three main components:

D To avoid flows contention near propose different flows gathering to a certain MR distant.

$>\quad$ In case of packet forwarding failure, use OR forwarding instead of packet direct retransmission.

$>\quad$ To reduce the overall delay of the $\mathrm{n}$ different streams, use forwarding of packets belonging to the $\mathrm{n}$ streams.

\section{A. Flow Gathering Construction (FGC)}

The main aim of FGC is to design an optimal throughput sub graph that avoids multiple throughput concentration. For each source VCS search the shortest path, assume the maximum and minimum source path length to lmax and lmin, respectively. Search the shortest path length of lag satisfies the following conditions:

$\cdot \operatorname{lag} \_\operatorname{lmin}$

- Path length 1 of a VS satisfies 1 lmax - lmin

If have satisfying the above two conditions, with greatest lag is elected. If there is no elected MR for the $\mathrm{n}$ VSs, search for any of $n-1$ VSs to continue the recurrence over the rest of MRs. If have no elected the algorithm switches to the SPR construction.

\section{B. Adaptive Frame length Control}

This scheme is implemented at the data link layer in the network stack. It is based on the following motivation. The probability of bit-error for transmission across channels is significantly high. In such scenarios, high packet corruption rate leads to retransmission of entire packets, leading to wastage of bandwidth. Further, it is important to note that the probability of packet error increases with the packet length. As the packet length increases, the probability of retransmission increases, with loss of efficiency.

\section{Dynamic Adaptive Segmentation}

Dynamic Adaptive Segmentation is an application layer technology with as the underlying transport layer mechanism. This scheme is designed to stream video by dynamically switching the streams according to the bandwidth available to the user [15]. This technology has several drawbacks as described below. Dynamic adaptive segmentation requires multiple bit-rate video sequences already encoded and stored at the server side and hence cannot be used in live broadcast video segmentation scenarios.

Further, the storage of video sequences at several rates tremendously increases the storage space required at the segmentation server. Since it is designed to wait for the next key frame, if the rate of the fading wireless channel is lowered momentarily, the playback at the client is halted, leading to jitter in the received video. Moreover, it switches between different bit-rate video sequences based on bandwidth, whereas the key criterion in wireless channel is packet corruption resulting from burst errors and not network congestion. Hence, the efficiency of bandwidth utilization will degrade significantly leading to lower quality video at the user end.

\section{Adaptive Bit rate Segmentation:}

Adaptive bit rate segmentation is a technique used in segmentation multimedia over computer networks. While in the past most video segmentation technologies utilized segmentation protocols such as Real Time Segmentation Protocol (RTSP), today's adaptive segmentation technologies are almost exclusively based and designed to work efficiently over large distributed networks such as the Internet.

It works by perceiving a user's bandwidth and CPU dimensions in real time and adjusting the quality of a video stream consequently. It involves the use of an encoder which can encode a single source video at manifold bit rates. The player client adjustments between subdivision the different encodings contingent on available resources. "The outcome: very little defending, fast start time and a good knowledge for both high-end and low-end influences." 
More explicitly, and as the implementations in use today are, adaptive bit rate separation is a method of video segmentation where the source content is prearranged at multiple bit rates, then each of the dissimilar bit rate streams are segmented into small multi-second portions. The subdivision client is made aware of the obtainable streams at differing bit rates, and sections of the streams by an obvious file.

\section{OVERVIEW OF PROTOCOL AND VIDEO}

Protocol based congestion control is especially suited for standard coaxial cable or fiber based networks, which have higher reliability compared to fading wireless links multiplexed over the shared frequency spectrum as explained in the previous section. As a result, packet error rate over a wireless link is meaningfully higher due to the burst error typical of the fading channel.

As shown in Fig.1, application of the 2-D Haar wavelet transformation [11] on the target video frame leads to a hierarchical base and enhancement layer decomposition of the frame. On completion of all the above phases, the encoded video base layer comprises of the most significant video information and is transmitted over the TCP connection of lowest index at a higher SNR and the enhancement layer over the second TCP connection at lower SNR. At the receiver, on successful reception of the video frame packets, the video decoder repeats the above stages in reverse order to rebuild the original video frames using the received video layers. The proposed application employs a sequential number for each TCP connection so that the receiver is aware of all the packets that have been lost due to corruption. Towards this end the application layer sets a field for the start of each frame.

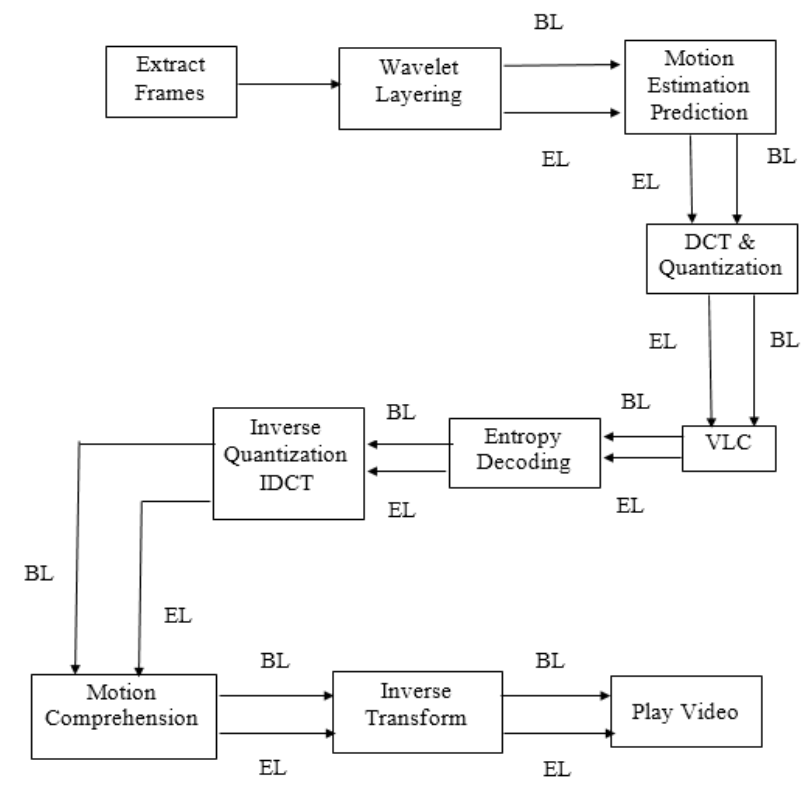

Fig 1. Video transmission over hetrogenety networkwith adaptive bitrate segmentation algorithm.

EL-Enhancement Layer, BL-Base Layer

\section{EXPERIMENTATION AND RESULTS}

From the data one can observe that using as the number of VSs increases, the streams delay increases dramatically. But with proposed algorithm the video delivery increases slightly from $0.6 \mathrm{~s}$ to $1 \mathrm{~s}$. From the two observe that when the number of VSs and the average hop length exceed 2, the scheme causes a decrease in the aggregate throughput because more used links contend with each other. But adaptive bit rate segmentation reduces contention between paths.

Therefore adaptive bit rate segmentation produces an advantage to increase the average throughput. The fig.2, also show that an increase in the number of VSs and the average hop length does not affect the throughput of adaptive bit rate segmentation. It shows the variation of priority route for segmentation video throughout the network. As the bit rate increases the flow under starts to suffer from major packet loss. But, because adaptive bit rate segmentation can increase the capacity of the network or can correct packet loss, adaptive bit rate segmentation reduces losses due to the delay and link packet loss in the network. 


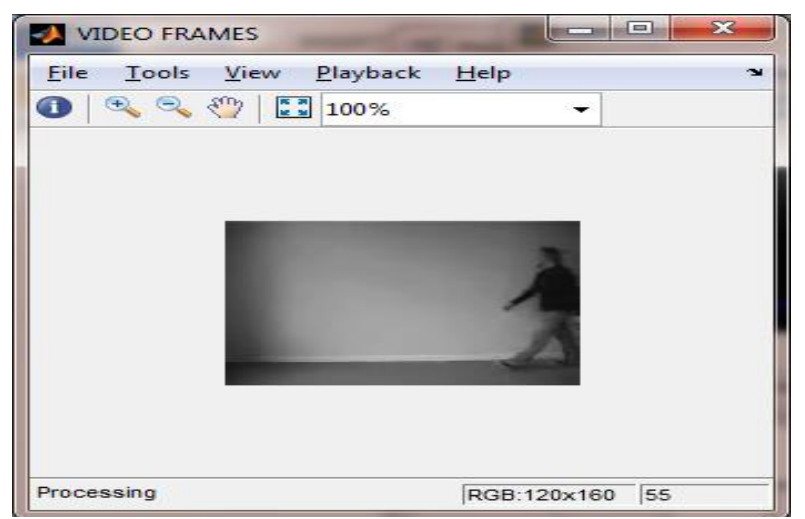

Fig 2. Frames from the video for processing segmentation

\section{CONCLUSION}

We have proposed a novel and well-organized layered adaptive bit rate segmentation based approach to subdivision the primary object in videos. This approach also uses innovative mechanisms to calculate the 'objectless' of a region and to compute similarity between object applications across mounts. The proposed approach outstrips the state of the art on the well-known dataset. It explicitly activities the common fate phenomenon exhibited by the different fragments of the forefront across multiple frames and numerous videos, making full use of the evidence available in the video cosegmentation setting but not available in other separation or co-segmentation location. With an evolving research area there are many issues to be solved in the area of multimedia segmentation. It seems that at present there is no single method exists that can act as the standard for multimedia segmentation. An algorithm that can solve all the problems of congestion control in segmentation applications is still in the stage as castle in the air. More research work is needed to solve these issues efficiently. The future work is to develop a novel protocol that can reach the stage of maturity to be the standard protocol for media segmentation.

\section{REFERENCES}

[1] M. Wang, B. Ni, X.-S. Hua, and T.-S. Chua, “Assistive tagging: a survey of multimedia tagging with human-computer joint exploration,” ACM Computing Surveys, vol. 44, no. 4, 2012.

[2] E. S. Spelke, "Principles of object perception," Cognitive Science, vol. 14, pp. 29-56, 1990.

[3] C. Fowlkes, D. Martin, and J. Malik, "On measuring the ecological validity of local figure/ground cues," in ECVP, 2003.

[4] M. Maire, "Simultaneous segmentation and figure/ground organization using angular embedding," in ECCV, 2010.

[5] X. Ren, C. Fowlkes, and J. Malik, "Figure/ground assignment in natural images," in ECCV, 2006.

[6] J. Stahl and S. Wang, "Convex grouping combining boundary and region information," in ICCV, 2005.

[7] Y. Lee, J. Kim, and K. Grauman, "Key-segments for video object segmentation,” in ICCV, 2011.

[8] D. Zhang, O. Javed, and M. Shah, "Video object segmentation through spatially accurate and temporally dense extraction of primary object regions," in CVPR, 2013.

[9] D. Sun, J. Wulff, E. B. Sudderth, H. Pfister, and M. J. Black, "A fully connected layered model of foreground and background flow," in CVPR,2013.

[10] T. Brox and J. Malik, "Object segmentation by long term analysis of point trajectories," in ECCV, 2010.

[11] P. M.A., Low-level and high-level contributions to figure-ground organization. Oxford University Press, 2014.

[12] M. Peterson and B. Gibson, "Must figure-ground organization precede object recognition? an assumption in peril," Psychological Science,vol. 5 , no. 5, pp. 253-259, 1994

[13] P. Ochs and T. Brox, "Object segmentation in video: A hierarchical variational approach for turning point trajectories into dense regions, "in ICCV, 2011.

[14] M. Keuper, B. Andres, and T. Brox, "Motion trajectory segmentation via minimum cost multi cuts," in ICCV, 2015.

[15] B. Alexe, T. Deselaers, and V. Ferrari, "What is an object?" in CVPR,2010. 